\section{Novel mutation of the notch3 gene in arabic family with CADASIL}

\author{
Saeed Bohlega \\ Department of Neurosciences, King Faisal \\ Specialist Hospital and Research Centre, \\ Riyadh, Saudi Arabia
}

\begin{abstract}
Mutations in the NOTCH3 gene are responsible for cerebral autosomal dominant arteriopathy with subcortical infarcts and leukoencephalopathy (CADASIL), an adult onset hereditary angiopathy leading to ischemic stroke, vascular dementia and psychiatric disorders. All mutation of NOTCH3 described so far are striking stereotyped leading to the gain or loss of cystiene residue in a given epidermal growth factor (EGF), like repeat. We report an Arabic family affected with CADASIL mutation, G1790 C, in Exon 11 of the NOTCH3 gene. This is the first novel mutation reported in Arabic CADASIL patients. This finding confirms that mutations in NOTCH3 are associated with the pathogenesis of CADASIL across different ethnic background.
\end{abstract}

\section{Introduction}

CADASIL is an autosomal dominant vascular disorder clinically characterized by a variety of symptoms including migraine with aura, mood disorder, vascular dementia, ischemic stroke and premature death. ${ }^{1-3}$ The vascular lesions underlying CADASIL are a non-arteriosclerotic, non-amyloid arteriopathy affecting small cerebral arteries with accumulating of granular and osmiophilic materials within the smooth muscles cell basement membrane.., 5 This initial thickening and expansion of the extracellular matrix can be found to a lesser extent in extra cerebral arteries such as skin arterioles. ${ }^{5}$

Mutation in the NOTCH3 gene is usually linked to CADASIL. ${ }^{6}$ NOTCH3 encodes a $300-k d$ transmembrane protein with a receptor and cell signal transduction function. Mutations are in majority mis-sense mutations causing the loss or gain of a cysteine residue and are detected in $95 \%$ of patients. ${ }^{7}$ All mutations are located within the epidermal growth factor (EGF) repeats in the extra cellular domain of the NOTCH3 gene and a strong clustering of the mutation were observed in Exon 3 and 4.8,9 Thus, there are more than 150 mutation have been identified. (The human gene mutation database www.hgmd.org), CADASIL is far more common than previously perceived and it may often be misdiagnosed because it can present under various guises. ${ }^{10}$ It also has been described in most ethnic groups from Western \& Central Europe, Japan, Turkey, South Africa and South East Asia ${ }^{11,12}$ and only few reported families from Arabic origin. 13,14 Hence, we described for a first time, a native Arabian family with CADASIL which harbor a novel mutation in NOTCH3 genes.

\section{History}

The family is a native Arabian family of Bedouins (Nomads) origin settled in NorthEastern part of the Arabian peninsulas.

The proband is a male seen at age of 37 years with 2 years history of recurrent throbbing headache with visual aura, and four months history repeated attacks of numbness in left side. These attacks suggestive of TIA, he is smoker, otherwise, no systemic disorder. His neurological examination was not revealing. His mother died at age 52 after 7 years history of repeated strokes and dementia (Figure 1). One brother had 2 strokes at age 35 years. He was depressed with hyperreflexia and right hemiparesis. Maternal grandfather also died at mid fifties with history of strokes and dementia. One younger sister suffered from migraine started at age 32 years and her neurological exam was normal. None had hypertension or diabetes and all blood test and cardiac evaluations were normal.

MRI brain of the index a case and his brother, sister and mother showed multiple deep white matter lesions (Figure 2) in temporal and periventricular regions (A-D) and in basal ganglia, thalami and pons (E\&F).

\section{Materials and Methods}

Genomic DNA was extracted from peripheral blood using an AutoGenFlex STAR automated DNA extractor (AutoGen, Inc.) Exons of the NOTCH3 gene reported to contain disease causing mutations (i.e., exons 2-5, 8, 11, 14, $18,19,22$ and 23 , were amplified by the polymerase chain reaction (PCR) and screened for mutations using dHPLC (WAVE; Transgenomic, Inc.) and Surveyor endonuclease digestion. Exons exhibiting altered elution were sequenced using a commercially available kit (A 1) and di-deoxy terminators. Sequences were analyzed on an automated DNA sequencer (ABI) and compared to normal sequence.

[Neurology International 2011; 3:e6]
Correspondence: Saeed Bohlega, MBC 76, Department of Neurosciences, King Faisal Specialist Hospital and Research Centre, PO Box 3354, Riyadh 11211, Saudi Arabia.

Tel. 966.505477665 - Fax: 966.1.442.4763.

E-mail: sboholega@kfshrc.edu.sa

Key words: NOTCH3, CADASIL, mutation, Arabs.

Acknowledgement: Special thanks to Dr Khalid Abu-Amero who performed the control studies and to Imelda Iturrios for preparing the Manuscript.

Received for publication: 20 June 2011.

Revision received: 7 June 2011.

Accepted for publication: 14 June 2011.

This work is licensed under a Creative Commons Attribution NonCommercial 3.0 License (CC BYNC 3.0).

(C) Copyright S. Bohlega., 2011

Licensee PAGEPress, Italy

Neurology International 2011; 3:e6

doi:10.4081/ni.2011.e6

\section{Results}

Affected individuals were heterozygous for a $\mathrm{G}$ to $\mathrm{C}$ mutation at nucleotide 1790 of the NOTCH3 gene (c.1790G $>$ C) that changes a codon for cysteine (TGC) to one for serine (TCC) at amino acid position 597 (p.Cys597Ser). This mutation was not detected in 120 ethnically related controls.

\section{Discussion}

The family described in this report showed various characteristics that are typical of CADASIL, an autosomal dominant pattern, recurrent ischemic stroke, migraine, cognitive impairment and premature death within 10 years after onset. One patient presented with late onset migraine with aura had an abnormal brain MRI, a feature along with family history is suggestive of this diagnosis. ${ }^{2}$ Positive brain MRI finding in asymptomatic individual indicate that NOTCH3 signaling may start up to a decade prior to clinical presentation. ${ }^{3}$

The C597S, NOTCH3 mutation in this family is likely the causative mutation as its co-segregate with the clinical phenotype and its absence in 120 ethnically related control. Furthermore, C597S is highly evolutionary conserved in the NOTCH3 gene, located in Exon $11^{5}$ and predict change of cysteine to one of serine at amino acid position 597 (nucleotide 1790 of the NOTCH3 gene (c1790G $>$ C). The loss of cysteine residue may 

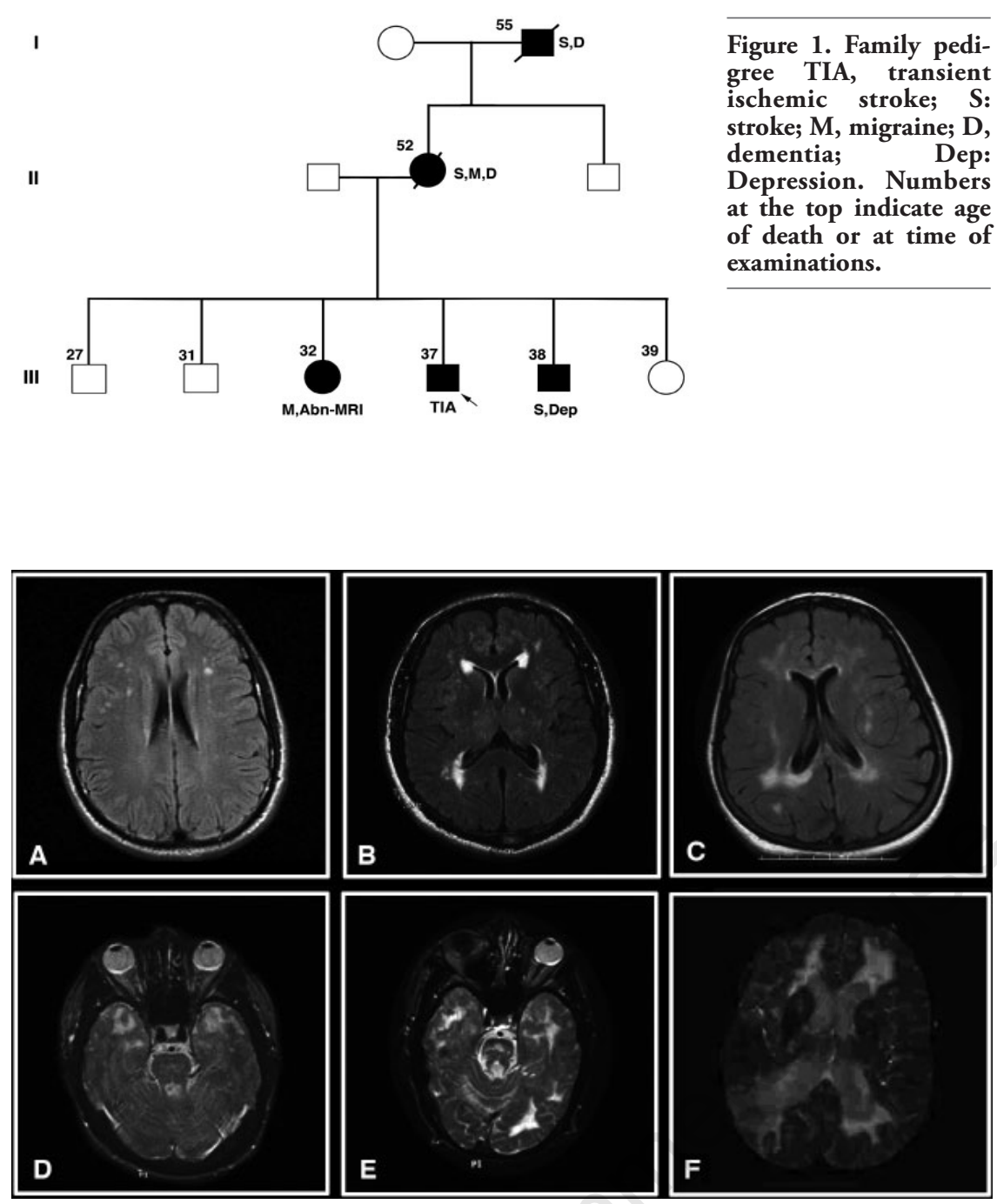

Figure 2. Representative magnetic resonance imaging scans from family members. (A) From sister (III: 32 in Figure 1). Note multiple lacunar lesions in deep white matter. (B) (Index case III-37). Note multiple periventricular ischemic lesions C \& D (from patient III-38). Scans (E \& F) (From mother II-52) showing different ischemic lesions in brain and brain stem.

disturb the di-sulfate bond in EGF-like repeat at EGF-14 domain.6,12 In patients with suggestive clinical and MRI findings of CADASIL necessitate either genes testing and/or electron microscopy or immuno-histochemical examination of a skin biopsy. Mutation of a pathogenic NOTCH3 gene gives the definite diagnosis but the great number of different mutations ( $>150)$ makes the search for less common mutation cumbersome. The most efficient strategy in some populations will depend in the patient's family history, founder mutations or the mutational background in

Figure 1. Family pedigree TIA, transient ischemic stroke; $S$ : stroke; M, migraine; D, dementia; Dep: Depression. Numbers at the top indicate age of death or at time of examinations.
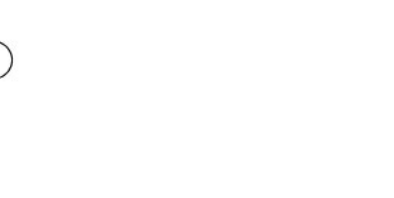